\title{
BMJ Open Finding the right balance with participation in exercise and sport for individuals with multiple sclerosis: protocol for a pre and post intervention feasibility study
}

\author{
Moira Smith (D) , ${ }^{1}$ Gavin Williams, ${ }^{2}$ Ruth Barker ${ }^{3}$
}

To cite: Smith M, Williams G, Barker R. Finding the right balance with participation in exercise and sport for individuals with multiple sclerosis: protocol for a pre and post intervention feasibility study. BMJ Open 2020;10:e035378. doi:10.1136/ bmjopen-2019-035378

- Prepublication history and additional material for this paper are available online. To view these files, please visit the journal online (http://dx.doi. org/10.1136/bmjopen-2019035378).

Received 30 0ctober 2019 Revised 18 January 2020 Accepted 12 February 2020

Check for updates

(c) Author(s) (or their employer(s)) 2020. Re-use permitted under CC BY-NC. No commercial re-use. See rights and permissions. Published by BMJ.

${ }^{1}$ College of Healthcare Sciences, James Cook University,

Townsville, Queensland,

Australia

${ }^{2}$ School of Health Sciences, The University of Melbourne, Melbourne, Victoria, Australia

${ }^{3}$ College of Healthcare Sciences, James Cook University, Cairns, Queensland, Australia

Correspondence to

Ms Moira Smith;

moira.smith2@jcu.edu.au

\section{ABSTRACT}

Introduction Individuals with minimal disability from multiple sclerosis (MS) requested advice on finding the right balance, between too much and too little exercise, when participating in their choice of sport or exercise. To optimise exercise participation during the early stages of the disease, a flexible exercise participation programme (FEPP) has been developed. The FEPP is novel because it provides guidance and support for individuals with MS to participate and progress in their preferred sport or exercise. The primary objective was to assess the feasibility of the FEPP. The secondary objective was to assess the feasibility of a larger trial to demonstrate the efficacy of the FEPP.

Methods and analysis A stage I feasibility study of the FEPP, using a single group preintervention/postintervention design, will be conducted with 16 participants with minimal disability from MS (Expanded Disability Status Scale level of $0-3.5$ ). The 12-week FEPP will guide participants to independently participate in their preferred sport or exercise at a location of their choice. Exercise progression will be guided by individual energy levels and a weekly telephone coaching session with a physiotherapist. Participation in exercise or sport will be recorded in parallel with assessment of disease biomarkers (plasma cytokines interleukin (IL)-2, IL-4, IL-6, $\mathrm{IL}-10$, interferon (IFN)- $\gamma$ and tumour necrosis factor (TNF)), subjective vitality and high-level mobility. Acceptability of the FEPP will be assessed using a sequential explanatory mixed methods design where the findings of a participant survey will inform the interview guide for a series of focus groups.

Feasibility of a larger trial will be assessed via process, resources, management and scientific metrics. Progression to a larger trial will depend on the achievement of specified minimum success criteria. Ethics and dissemination Ethical approval has been obtained for this study from the James Cook University Human Research Ethics Committee (H7956). Dissemination of findings is planned via peer-reviewed journals, conference presentations and media releases. The protocol date was 21 December 2019, V.1.

Trial registration number The trial is registered with Australian New Zealand Clinical Trials Registry (ANZCTR), ACTRN12620000076976.

\section{Strengths and limitations of the study}

- The flexible exercise participation programme (FEPP) is a consumer-driven programme for individuals with multiple sclerosis.

- Consumer preference for sport or exercise mode is central to the FEPP.

- Active participation in exercise or sport is focused on participation rather than impairment.

- Study findings will inform the design of a larger trial.

- This study without a control group demonstrates feasibility rather than efficacy.

\section{INTRODUCTION}

Individuals with minimal disability from multiple sclerosis (MS), have recently reported participating in, or wanting to participate in, sport or high-level exercise, such as running, squash or road cycling. ${ }^{1}$ While their preference was to undertake their choice of sport/exercise independently at a time suitable to them, they also wanted advice on finding the correct balance between too much activity, which may exacerbate symptoms, and too little activity, which could unnecessarily limit participation. Yet few study participants had received any such advice, nor had they been given their choice of exercise. ${ }^{1}$ Commonly, exercise interventions for individuals with MS are provided in a clinical or home-based setting where the individual follows a prescribed activity or exercise programme. High-level mobility activities such as running, sport or outdoor leisure pursuits are not typically targeted. ${ }^{2}$ Instead, exercise interventions prescribed include progressive resistance training, balance training and stationary cycling, addressing impairments such as strength and balance. ${ }^{3-5}$ Functional outcomes are typically focused on walking ${ }^{67}$ with no attention to the benefits for 
high-level mobility, even for those who are higher functioning. ${ }^{2}$ Benefits of participation in regular sport and exercise according to the preference of individuals with MS have not been reported.

Empowering individuals with the autonomy to manage their own exercise prescription, according to their individual goals, is an important concept in exerting control over the impact of the disease. Previous interventions examined in clinical trials for MS have not commonly allowed for this diversity. Therefore, exploration of ways to adapt and modify personal exercise choices to improve or maintain participation is required. To address this need, a flexible exercise participation programme (FEPP) has been developed to offer individuals choice and to guide their mode and dose of exercise. The FEPP has been based on existing recommendations for general and advanced aerobic exercise for individuals with MS. ${ }^{8}$ It has also been informed by the guidelines for healthy individuals proposed by the American College of Sports Medicine and equivalent version by the Australian Government Department of Health, which aim to reduce the risk of chronic disease. ${ }^{910}$ The FEPP is a stepping stone supported pathway to move from low levels of aerobic exercise towards meeting or exceeding advanced aerobic exercise guidelines for individuals with MS. The aim of the FEPP is to assist individuals with minimal disability from MS in finding the right balance between too little and too much exercise, and to maximise the benefits of exercise for individuals with MS. The FEPP provides a personally tailored programme to achieve exercise participation goals specific to the individual and is guided by the individual's perceived energy levels. By monitoring and responding to energy levels, participants are using a pacing technique, which is a method for managing energy effectively, ${ }^{11}$ thereby enabling participation. The FEPP provides a framework for a graded response to exercise rather than an 'all-or-nothing' approach. Support is provided on a weekly basis, by a physiotherapist, using recognised behaviour change techniques to enable individuals to vary their physical activity. ${ }^{12}$ It is evident that individuals with MS need support to increase and maximise participation in their choice of exercise. ${ }^{1}$

Many individuals with mild to moderate disability from MS do not meet recommended levels of physical activity required to obtain fitness benefits ${ }^{13}$ despite guideline recommendations. ${ }^{814}$ For those who do, the guidelines provide a baseline for exercise, ${ }^{8}$ but the maximum safe dose is not yet known. ${ }^{14}$ Manipulation of the exercise dose is required to determine the optimum level, which maximises benefits and is free from negative consequences, for each individual with MS. Historically, concerns existed around the possibility of exercise increasing fatigue for individuals with MS. ${ }^{15}$ Even though evidence now suggests otherwise,${ }^{16}$ levels of fatigue continue to guide practice. ${ }^{14}$ In the FEPP, a shift away from such impairment-based assessment is proposed with assessment of perceived energy levels prior to exercise to guide selection of exercise dose. Energy conservation approaches for individuals with MS are important tools for planning and pacing activities in order to manage fatigue in daily life. ${ }^{11} 17$ Attention to available energy prior to exercise may enable an informed decision on whether to progress, maintain or regress exercise dose, and assist in finding the right balance between too much and too little activity. Measurement of vitality following a period of regular participation in exercise may also serve to demonstrate long-term benefits of exercise. ${ }^{18}$

Physiological benefits of exercise include improvements in aerobic capacity, balance and muscle strength. ${ }^{19-21}$ In addition, it has been proposed that exercise may have a neuroprotective and disease-modifying effect on MS. ${ }^{22}{ }^{23}$ Biomarkers that may serve as indicators of exercise-induced neural changes in MS include neurotrophic factors and cytokines. ${ }^{24}$ Neurotrophic factors can increase as a result of exercise, such as brain-derived neurotrophic factor, which has a role in neurogenesis and neuroprotection of the central nervous system. ${ }^{22}{ }^{25}$ Cytokine levels have also shown change following exercise in individuals with MS. ${ }^{26}{ }^{27}$ Cytokines assist in regulating the immune response. ${ }^{28}$ In MS, there is an imbalance between the levels of proinflammatory and anti-inflammatory cytokines, with higher levels of proinflammatory cytokines linked to the demyelination process. ${ }^{28}$ Reduction in proinflammatory cytokines can occur following exercise ${ }^{26}$ However, the evidence is inconsistent as to whether the change in cytokine profile is the mechanism for physiological improvement following exercise and hence requires further investigation. ${ }^{29}$ Furthermore, the effects of exercise dose (ie, frequency, intensity, duration and mode) on cytokine levels remains unknown. ${ }^{29} 30$

The purpose of this study was to assess the feasibility of the FEPP, a novel sport and exercise intervention for individuals with MS. Individuals with minimal disability from MS will be invited to participate in their preferred exercise. Response to exercise dose will be assessed using disease biomarkers, subjective vitality, as a measure of energy, high-level mobility, and subjective reporting. The objectives of the study were to

1. Assess the feasibility of the FEPP for individuals with minimal disability from MS.

a. Does the FEPP enable achievement of goals for participation in exercise and sport for individuals with MS?

b. What is the best method to describe and report on the exercise or sport intervention?

c. Is there a relationship between the level of participation in exercise and clinical/physiological outcomes?

- Plasma cytokine levels (IL-2, IL-4, IL-6, IL-10, IFN- $\gamma$ and TNF).

- Vitality (energy levels measured via the Subjective Vitality Scale).

- High-level mobility (measured via the High-Level Mobility Assessment Tool (HiMAT)) .

d. Is the FEPP acceptable from the perspective of individuals with MS? 
2. Assess the feasibility of a larger clinical trial against the following minimum success criteria:

a. No reports of serious adverse events as a result of completing the FEPP.

b. A minimum of $80 \%$ of participants able to modify exercise participation using the FEPP.

c. A minimum of $80 \%$ of participants report satisfaction with the FEPP.

d. A minimum of $20 \%$ attrition from the 12-week FEPP.

e. A minimum of $75 \%$ recruitment of the intended 16 participants.

f. A minimum of $75 \%$ completion of each outcome measure.

\section{METHODS AND ANALYSIS \\ Study design}

This stage I feasibility study will involve a single group preintervention/post-intervention design to explore implementation of a 12-week FEPP with individuals with minimal disability from MS. Participation in exercise or sport will be recorded in parallel with assessment of disease biomarkers, subjective vitality and high-level mobility.

Acceptability of the FEPP to participants will be assessed using a sequential explanatory mixed methods design. ${ }^{31}$ Perceived effective/ineffective elements of the FEPP and potential adaptations will be explored to guide refinement of the FEPP. Assessment of feasibility metrics (process, resources, management and scientific) will inform the suitability of a larger trial.

\section{Study setting}

Data collection will occur in the James Cook University (JCU), Australia, in January 2020. The intervention will occur according to each participant's preferred mode of exercise and preferred setting, for example, sports centre, gym or outdoor pursuit in his/her local environment.

\section{Participants}

Individuals with MS who meet the following inclusion criteria will be invited to participate: (1) diagnosis of relapsing remitting MS as defined by the $2017 \mathrm{McDonald}$ criteria $^{32}$; (2) independent mobility as defined by Expanded Disability Status Scale level 0-3.5 ${ }^{33}$; (3) stability, that is, not worsening in the past 3 months on diseasemodifying drugs (e.g. alemtuzamab, natalizumab and ocrelizumab $)^{34}$; (4) 18 years of age or over; and (5) ability to provide informed consent. Potential participants will be excluded if they have (1) any concomitant neurological condition or (2) an additional health condition that would prohibit their participation in aerobic exercise or sport.

\section{Recruitment}

Participants will be recruited via (1) media: television, newspaper and social media; (2) flyer distributed by MS Queensland and by consultant neurologists; (3) flyer displayed in community settings (eg, community notice boards and medical practices; (4) JCU website and social media; and (v) snowballing. Potential participants will be advised to contact the primary researcher by email or telephone for further information. Once contacted, the primary researcher will screen potential participants in person or via telephone against the inclusion/exclusion criteria.

All potential participants who meet the eligibility criteria will be provided with an information letter and a consent form, either electronically or via post with a replypaid envelope, according to their preference. Those who wish to participate will be advised to return the signed consent form in person, electronically or via post. Participants can withdraw from the study at any time without explanation or prejudice.

\section{Sample size}

Sixteen participants will be recruited, allowing for a $25 \%$ dropout rate. A sample size of 12 participants has been recommended for feasibility studies. ${ }^{35}$ As this study is designed to assess the feasibility of a larger trial, a formal sample size calculation will not be required.

\section{Intervention}

All 16 participants will undertake the FEPP, a 12-week programme, in which participants choose their preferred mode of exercise as well as the time and location for exercise. Exercise will be performed independently by the participant (ie, not supervised by the research team). The FEPP is illustrated in flowchart format in figures 1 and 2. The FEPP flowchart will guide the participant to incrementally progress, maintain or regress their activity level based on performance feedback. The FEPP has two streams (table 1) to enable progression of activity level relative to the individual's baseline activity level.

Stream 1 is for participants who do not meet the MS general aerobic exercise guidelines of at least $30 \mathrm{~min}$ of moderate intensity aerobic exercise three times per week. ${ }^{8}$ Moderate intensity exercise is defined as $40 \%-59 \%$ of heart rate reserve and can be scored as $12-13$ on a 6-20 rating of perceived exertion (RPE) scale. ${ }^{9}$ Participants progress through the stream modifying frequency and duration of exercise, as guided by the FEPP, until they reach the MS general aerobic exercise guidelines. ${ }^{8}$ Participants can opt to maintain this activity level for the remainder of the programme if they are satisfied with their participation in their chosen sport or exercise in accordance with their goals. Alternatively, participants can progress through stream 2.

Stream 2 is for participants who meet MS general aerobic exercise guidelines. This stream is designed to incrementally progress exercise towards meeting or exceeding the MS advanced aerobic exercise guidelines. ${ }^{8}$ These guidelines recommend an exercise duration approaching $40 \mathrm{~min}$; frequency approaching 5 days per week and intensity approaching 15 on an RPE scale of 6-20 points. ${ }^{8}$ Participants progress through the stream by modifying frequency, intensity and duration of exercise 


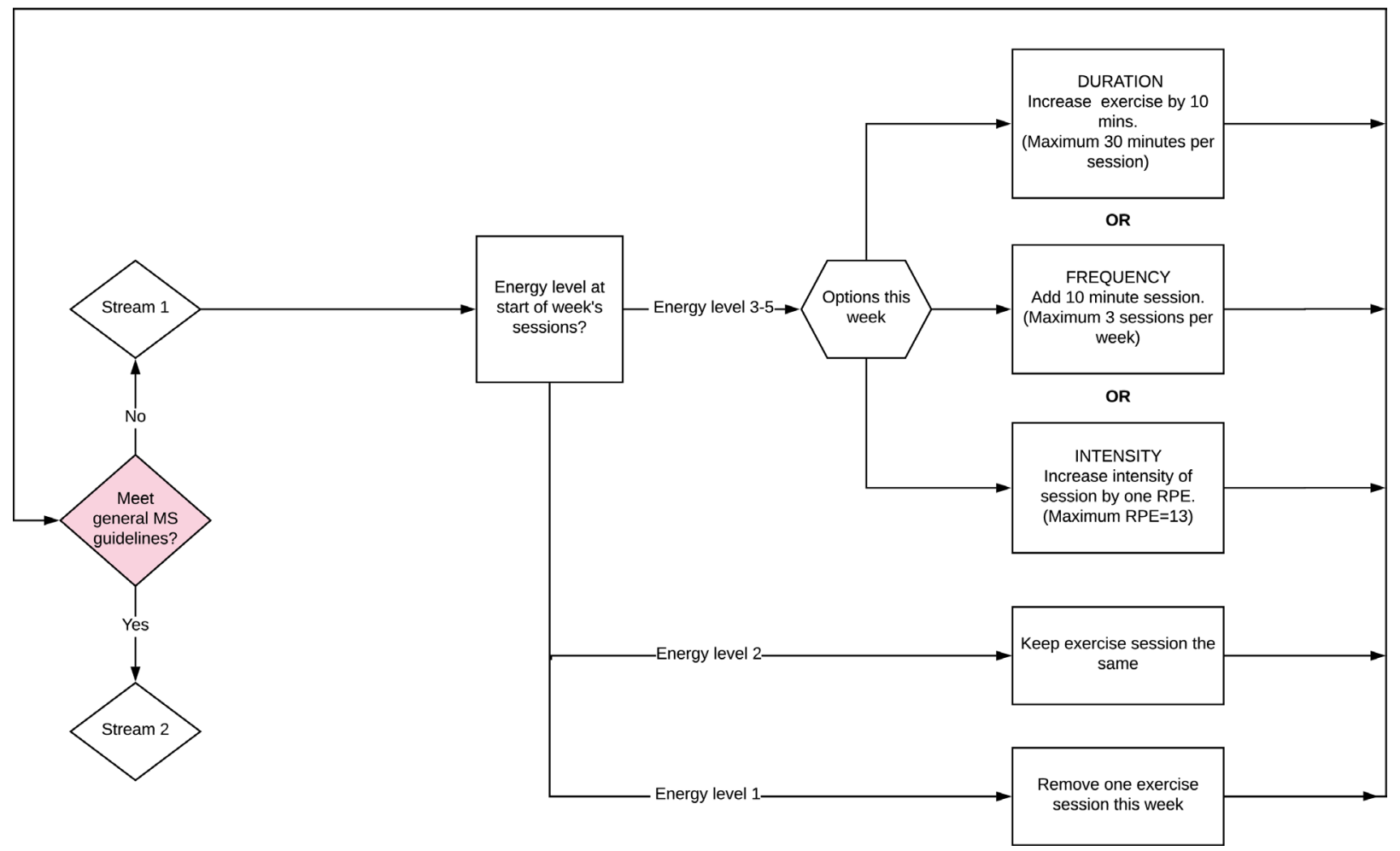

Figure 1 FEPP stream 1.

until they are satisfied with their participation in their chosen sport or exercise in accordance with their goals. This may be below, at or above MS advanced aerobic exercise guidelines. Participants continue with their optimum participation for the duration of the programme.

Each participant will begin the FEPP with an individual interview conducted by a physiotherapist (MS) to identify and discuss their goals for participation in sport or exercise. The participant determines their mode of exercise or sport, whether performed individually, with others or as part of a team, and indoors or outdoors. This information will be recorded in the participant database and exercise diary. Exercise progression will be guided by the FEPP. The FEPP stream allocation will be determined by the participant's baseline activity level recorded on entry to the programme.

Progression through the FEPP for both streams will be determined by the participant's rating of perceived energy levels over the course of each week. A single question, 'How

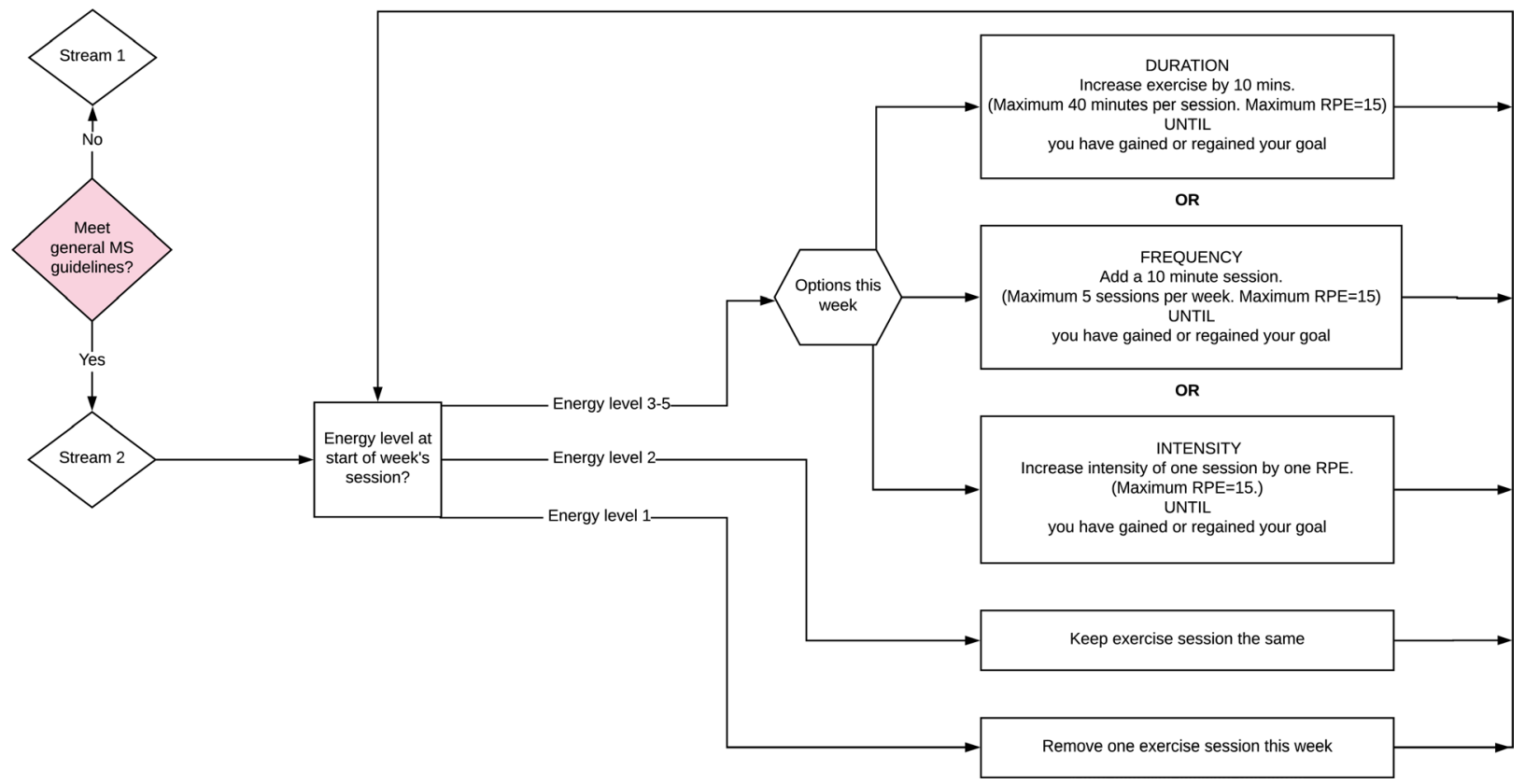

Figure 2 FEPP stream 2. 


\begin{tabular}{llll}
\hline & Current aerobic exercise & Intervention & Outcome \\
\hline $\begin{array}{l}\text { Stream 1 } \\
\begin{array}{l}\text { Does not meet MS general } \\
\text { aerobic exercise guidelines }\end{array}\end{array}$ & $\begin{array}{l}\text { Less than 30 min moderate } \\
\text { intensity three times per week }\end{array}$ & FEPP stream 1 & $\begin{array}{l}\text { MS general aerobic exercise guidelines are } \\
\text { achieved, with progress to stream 2. }\end{array}$ \\
$\begin{array}{l}\text { Stream 2 } \\
\begin{array}{l}\text { Meets MS general aerobic } \\
\text { exercise guidelines }\end{array}\end{array}$ & $\begin{array}{l}\text { 30 min or more of moderate } \\
\text { intensity three times per week }\end{array}$ & FEPP stream 2 & $\begin{array}{l}\text { Exercise participation goals are satisfied, } \\
\text { which may be below, at or above MS } \\
\text { advanced aerobic exercise guidelines. }\end{array}$ \\
\hline
\end{tabular}

FEPP, flexible exercise participation programme; MS, multiple sclerosis.

would you rate your overall energy levels this week?', will be scored by participants using the Energy Monitoring Tool, a 5-point energy Likert scale ranging from no energy to maximum energy (figure 3). Single-item questions such as this are used commonly to provide a quick response to self-rated health status. ${ }^{36}$ The Energy Monitoring Tool will guide incremental progressions or regressions using manipulation of frequency, intensity, time and type of exercise, that is, the FITT principle of exercise prescription, ${ }^{9}$ as indicated on the FEPP (figures 1 and 2).

Prior to and throughout the 12-week period, participants will be supported to participate in exercise or sport via a coaching session with a physiotherapist, once each week, via telephone. Behaviour change techniques known to assist with participation in exercise and sport ${ }^{12}$ will be used as listed in table 2, together with their definition ${ }^{12}$ and planned application.

\section{Outcome measures and data collection}

Data collection will take place via face-to-face visits, telephone interviews, email or post. Outcome measurement will occur face-to-face at JCU, Australia. The timeline for data collection of each outcome measure is displayed in table 3 .

\section{Feasibility outcomes}

The primary objective of the study was to assess the feasibility of implementing the FEPP for individuals with MS, in accordance with stage I feasibility trials specific to MS. ${ }^{37}$ Process, resources, management and scientific feasibility outcomes will be assessed. Process measures will include participant recruitment, eligibility, refusals, retention and attrition. Resources and management refer to the administrative aspects of the study such as data entry, finance and communication time with participants and staff. Scientific feasibility outcomes address aspects of safety, adverse events, compliance and potential treatment effects. This process of recording feasibility metrics has been used in other feasibility studies with MS populations. ${ }^{38} 39$

\section{Clinical outcomes}

Clinical outcomes will include the three domains of the International Classification of Functioning, Disability and Health framework, ${ }^{40}$ which are body structures/functions, activities and participation.

\section{Primary outcome}

\section{Participation}

The primary clinical outcome is participation goals in sport and exercise according to the participant's choice measured by the Goal Attainment Scale (GAS) ${ }^{41}{ }^{42}$ The GAS measures goal achievement (positive or negative) on a 5-point scale and can be quantified as a single aggregated goal attainment score for analysis. ${ }^{42}$ The GAS is a responsive measure for individuals with $\mathrm{MS}^{43}$

During the preintervention interview with the primary researcher, participants will be asked to identify their goals for participation in exercise and sport. The participant will be guided to set specific, measurable, achievable, relevant and timed (SMART) goals, ${ }^{44}$ for example, to cycle to work three times per week by the final 2 weeks of the FEPP. One to three goals will be set to represent the participant's key priorities. ${ }^{42}$ Reassessment of goals by the

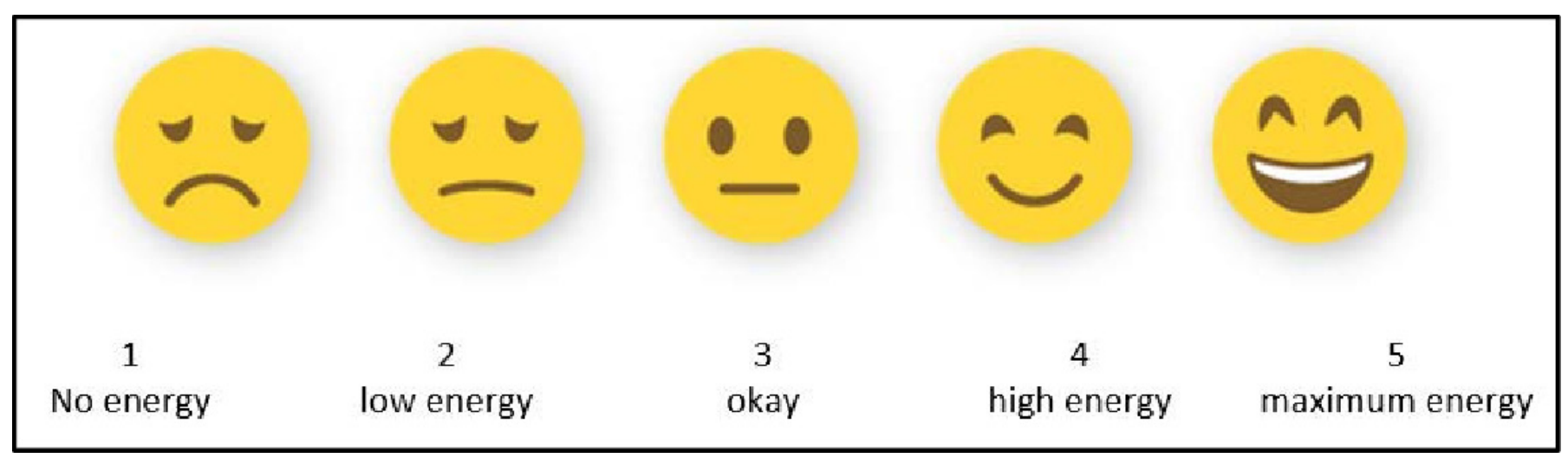

Figure 3 Energy Monitoring Tool. 
Table 2 Behaviour change techniques, definitions and application framework

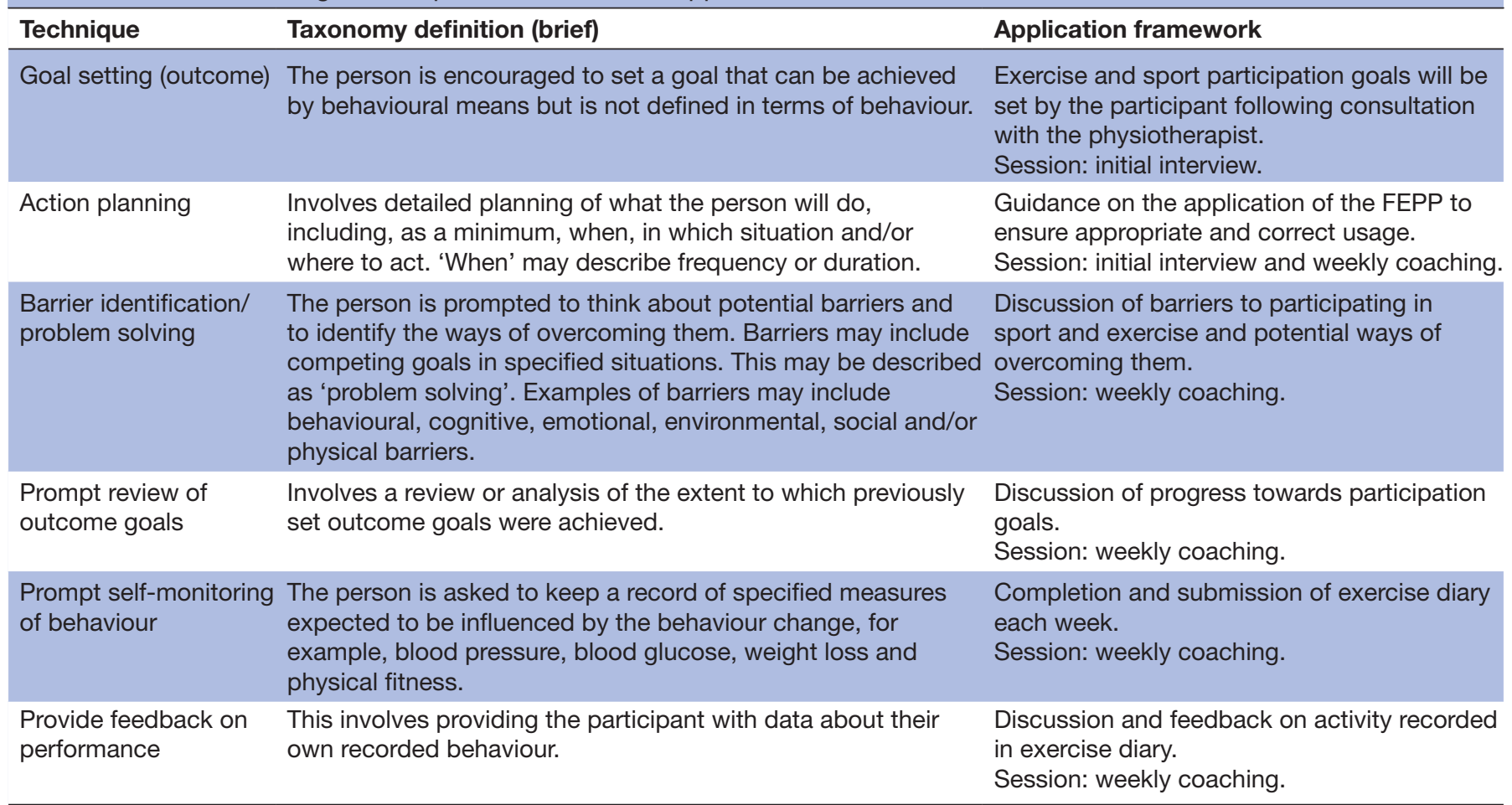

FEPP, Flexible exercise participation programme.

participant will take place during the postintervention interview with the primary researcher.

\section{Secondary outcomes}

Body structures and function: plasma cytokines

To identify the effects of exercise on cytokines, a $4 \mathrm{~mL}$ blood sample will be collected from each participant via pathology services during the week preintervention and postintervention, which is in accordance with similar studies. $^{26} 2745$ Blood samples will be collected between 08:00 and 09:30, following an overnight fast of at least 10 hours. Samples will be collected in the morning to prevent any circadian changes in gene expression and to allow for a more meaningful comparison. ${ }^{46}$ Blood will be collected in $4 \mathrm{~mL}$ EDTA vacutainers. Following collection, blood samples will be chilled and immediately transferred to the JCU Molecular and Cell Biology Department for processing. The samples will be centrifuged, plasma collected and stored at $-80^{\circ} \mathrm{C}$ until all samples are ready for analysis. Cytokine levels (IL-2, IL-4, IL-6, IL-10, IFN- $\gamma$ and TNF) will then be tested, following manufacturer's instructions, using the commercially available kit: BD Cytometric Bead Array (CBA) Hu Th1/Th2 Cytokine Kit II.

\section{Body functions: vitality}

Perceptions of vitality will be self-reported by participants using the six-item version of the Subjective Vitality Scale, which has been validated for use with the general population $^{47}$ and has previously been used with the MS population. ${ }^{48}$ The Subjective Vitality Scale assesses the experience of being full of energy and alive, via six questions rated on a 7-point scale from 'not at all true' to 'very true', and provides an overall score of participants' energy. ${ }^{47}$ The primary researcher will collect these data at four time periods across the study: baseline and 4, 8 and 12 weeks (completion) via face-to-face or telephone interview.

\section{Activities: high-level mobility}

High-level mobility (ie, running or jumping) will be measured using the HiMAT ${ }^{49}$ to explore the relationship between high-level mobility and participation in exercise for individuals with MS. The HiMAT assesses high-level mobility across 13 items, such as running, jumping and climbing stairs, with a total point score of 54 and higher scores indicating higher levels of mobility. The HiMAT is a valid and reliable tool for assessing high-level mobility. ${ }^{50}$ A physiotherapist, who is independent of the intervention that has been trained in the use of the HiMAT, will assess the participants during the 1-week preintervention and postintervention period.

\section{Participation}

Participation in sport or exercise during the intervention period will be measured using an exercise diary. The participant will record the frequency, intensity, time and type of exercise undertaken each week in an electronic exercise diary and email it to the primary researcher on a weekly basis. Where participants do not have access to email, a paper format will be provided, together with a reply-paid envelope. FITT data will provide a record 
Table 3 Data collection and outcome measures

\begin{tabular}{|c|c|c|c|c|c|}
\hline Outcome measures & & Collection procedure & $\begin{array}{l}\text { Baseline } \\
\text { evaluation }\end{array}$ & $\begin{array}{l}\text { During } \\
\text { intervention }\end{array}$ & $\begin{array}{l}\text { Post intervention } \\
\text { evaluation }\end{array}$ \\
\hline \multicolumn{6}{|l|}{ Feasibility measures } \\
\hline Process & $\begin{array}{l}\text { Recruitment. } \\
\text { Eligibility. } \\
\text { Refusals. } \\
\text { Retention. } \\
\text { Attrition. } \\
\text { Adherence. }\end{array}$ & $\begin{array}{l}\text { Documentation of } \\
\text { All contacts with potential participants. } \\
\text { Participant flow through study. } \\
\text { Adherence via exercise diary. }\end{array}$ & $\sqrt{ }$ & Daily & $\sqrt{ }$ \\
\hline Resources & $\begin{array}{l}\text { Communication. } \\
\text { Finance. }\end{array}$ & $\begin{array}{l}\text { Documentation of } \\
\text { Duration and frequency of } \\
\text { communication between participants/ } \\
\text { staff (email, telephone and face-to-face } \\
\text { contact). } \\
\text { Communication difficulties. } \\
\text { All costs associated with the study. }\end{array}$ & $\sqrt{ }$ & Daily & $\sqrt{ }$ \\
\hline Scientific & $\begin{array}{l}\text { Safety. } \\
\text { Adverse events. } \\
\text { Compliance. } \\
\text { Treatment effect. }\end{array}$ & $\begin{array}{l}\text { Documentation of adverse and serious } \\
\text { adverse events } \\
\text { Via exercise diary. } \\
\text { Via weekly check-in with physiotherapist. } \\
\text { Via reporting safety concerns and } \\
\text { adverse events as per university policy. } \\
\text { Documentation of compliance } \\
\text { Via exercise diary and weekly check-in. } \\
\text { Treatment effect } \\
\text { Documentation of clinical outcome } \\
\text { measures preintervention/ } \\
\text { postintervention. }\end{array}$ & $\sqrt{ }$ & Daily & $\sqrt{ }$ \\
\hline
\end{tabular}

\section{Participation outcome}

\begin{tabular}{|c|c|c|}
\hline Goal attainment scale & Face-to-face or telephone data collection & $\sqrt{ }$ \\
\hline Cytokines & $\begin{array}{l}\text { Collection at the James Cook University } \\
\text { pathology site }\end{array}$ & $\sqrt{ }$ \\
\hline Subjective Vitality Scale & Face-to-face or telephone data collection & $\begin{array}{l}\text { Weeks } 4 \\
\text { and } 8\end{array}$ \\
\hline HIMAT & Face-to-face assessment and data collection $\sqrt{ }$ & $\sqrt{ }$ \\
\hline \multicolumn{3}{|c|}{ Subjective acceptability outcomes } \\
\hline Participant survey & Electronic data collection & $\sqrt{ }$ \\
\hline Focus group interviews & Face-to-face recorded interviews & $\sqrt{ }$ \\
\hline
\end{tabular}

of change in aerobic activity across the duration of the study. Specifically, comparisons will be made on a weekby-week basis as to whether participants meet or exceed MS general and advanced aerobic exercise guidelines.

\section{Acceptability of the FEPP}

Participant survey

A participant survey will provide an initial assessment of the acceptability of the intervention to the participants. Three key areas (satisfaction, usability and suitability) will be explored in a short survey using a 5-point Likert scale (online supplementary file) based on similar surveys used with individuals with MS. ${ }^{38} 51$ The survey will be provided electronically to each participant on completion of the study via the survey platform Qualtrics. ${ }^{52}$ If participants are unable to access the survey electronically, it will be provided in paper format. Survey responses will remain anonymous.

\section{Focus group interviews}

Focus group interviews will take place during the 6-week postintervention period to gain greater insight into participants' perceptions of the FEPP than the survey alone. Question design will be based on participant survey results regarding acceptability and recommendations for improvement of the FEPP. In addition, the focus groups 


\begin{tabular}{|c|c|}
\hline $\begin{array}{l}\text { Data } \\
\text { collection } \\
\text { method }\end{array}$ & Questions \\
\hline $\begin{array}{l}\text { Participant } \\
\text { survey }\end{array}$ & $\begin{array}{l}\text { Acceptability of intervention: } \\
\text { Satisfaction. } \\
\text { Usability. } \\
\text { Suitability. }\end{array}$ \\
\hline \multirow[t]{6}{*}{ Focus group } & $\begin{array}{l}\text { Acceptability of intervention } \\
\text { Participant survey results will guide the } \\
\text { questions. }\end{array}$ \\
\hline & $\begin{array}{l}\text { Effectiveness of the FEPP } \\
\text { Changes in participation in physical activity, } \\
\text { exercise or sport during the programme. } \\
\text { Changes in energy levels during the } \\
\text { programme. } \\
\text { Changes in high-level mobility during the } \\
\text { programme. } \\
\text { Long-term changes in participation in physical } \\
\text { activity, exercise or sport. }\end{array}$ \\
\hline & $\begin{array}{l}\text { Goals } \\
\text { Goal setting. } \\
\text { Achievement /non-achievement of goals. }\end{array}$ \\
\hline & Finding the balance \\
\hline & $\begin{array}{l}\text { Plans to continue with physical activity, exercise } \\
\text { or sport }\end{array}$ \\
\hline & $\begin{array}{l}\text { Suggestions to improve the FEPP or the process. } \\
\text { Participant survey results will guide the } \\
\text { questions. }\end{array}$ \\
\hline
\end{tabular}

will explore the participants' perspectives on the effects of the programme. The focus group study will adopt an exploratory qualitative descriptive methodology in order to gain a rich description of participants' experiences of the FEPP and to produce authentic reporting of the participants' experience. ${ }^{53} 54$

All participants will be invited to attend the focus group interviews. Each group will contain a minimum of three and a maximum of six participants per group, depending on participant availability. Where participants are unable to attend a focus group interview, they will be offered a one-to-one interview.

Methods used to determine FEPP acceptability are outlined in table 4 .

\section{Data management}

On entry to the study, participants will be allocated a unique identifying code which will then be recorded on all datasets pertaining to that individual. The confidential coding system will be held in a file separate from the other datasets. All data will be stored on the primary researcher's computer, which is password protected. A secondary copy will be stored on a secure research storage platform. When in use, all data will be saved to the computer and backed up daily. On completion, data will be stored in the JCU institutional repository for a minimum of 15 years.

\section{Patient and public involvement}

A qualitative study on active participation in sport and exercise informed the development of this protocol. ${ }^{1}$ Participants with minimal disability from MS highlighted that they want to participate in their preferred exercise or sport at a time that suits them. Importantly, participants identified that they need assistance in determining the dose of exercise they should undertake. This is the premise for the current feasibility study.

\section{DATA ANALYSIS}

Data analysis will occur in accordance with the objectives of the study: to assess the feasibility of the FEPP for individuals with MS and to assess the feasibility of conducting a larger clinical trial.

\section{Feasibility data analysis}

Descriptive statistics will be used to report on the process, resources, management and scientific feasibility domains of this study. The process domain (eg, recruitment and retention) will inform the feasibility of achieving the sample size required for a larger trial. The resources and management domains will inform the financial and administrative requirements for a larger trial. The scientific domain will identify the suitability of the outcome measures and any risk management required for a larger trial. In addition, the scientific domain will provide preliminary data on the effect and acceptability of the FEPP for individuals with MS and hence the feasibility of the FEPP.

\section{Clinical data analysis}

Clinical outcomes will be analysed descriptively rather than through formal hypothesis testing, as is the nature of feasibility trial data. ${ }^{55}$ Change from pre-FEPP to postFEPP will be described based on the (1) GAS; (2) exercise frequency, intensity and duration of exercise; (3) Subjective Vitality Scale; (4) HiMAT; and (5) cytokine levels. Changes in cytokine levels will be analysed with conventional flow cytometry analysis software by gating on the appropriate bead clusters and measuring the phycoerythrin median value for the bound analyte.

\section{FEPP acceptability data analysis}

Participant survey responses will be analysed descriptively using frequency distribution, central tendency and dispersion. Focus group data will be analysed in accordance with the exploratory qualitative descriptive methodology. Following reading and rereading of the dataset, each line of data will be coded, using a short title or word enabling clear identification of topics within the data. ${ }^{56}$ Inductive thematic analysis will be used to analyse the patterns, with similar codes brought together to identify emergent themes from the bottom up. ${ }^{54}$ Themes will subsequently be reviewed to check that they work in relation to the coded extracts by checking and rechecking the data; analysis will continue until themes are refined 
and a thematic map is created. ${ }^{57}$ Codes and patterns from one focus group dataset will be reviewed by a secondary researcher to check and verify, or to identify error, as part of quality assurance. ${ }^{58}$ In addition, member checking will take place with one member from each focus group to ensure appropriate representation of participant experiences.

Interpretation of the data through thematic analysis will enable a well-organised descriptive evaluation of the FEPP from the perspective of the participants. The final analysis will involve exploration of how the focus group data explain the quantitative participant survey data in accordance with the sequential explanatory mixed methods design. ${ }^{31}$

\section{Data analysis summary}

Collectively, the data will provide a comprehensive analysis of the feasibility of conducting a larger trial to assess the effectiveness of using the FEPP with individuals with MS. Progression to a larger trial will be dependent on the logistics of implementing the trial (process and resource metrics), together with the feasibility of the FEPP. Feasibility of the FEPP will be dependent on participants' safety, ability to modify exercise prescription with minimal supervision, preliminary effectiveness and participant acceptability with the intervention. Progression to a larger trial will be dependent on achievement of specified minimum success criteria.

\section{Ethics and dissemination}

Ethical approval has been obtained for this study protocol from the JCU Human Research Ethics Committee (H7956). The research team will be briefed on the requirements for conduct of this study in accordance with the National Statement on Ethical Conduct in Human Research. $^{59}$

This novel approach to participation in exercise in sport has been guided and driven by individuals with MS who have minimal disability. This approach has the potential to empower individuals with MS to independently engage in and optimise their participation in exercise according to their own preferences. The results of this study will inform future research in finding the balance between too much and too little participation in exercise and sport, for people with MS. Dissemination of study findings is planned via peer-reviewed journals, national and international conferences and associated media releases.

Contributors MS is the principal investigator. MS, GW and RB conceived and designed the study. MS wrote the first draft of the manuscript with subsequent revisions from all authors. MS will lead the operation of the study with support from GW and RB.

Funding The authors have not declared a specific grant for this research from any funding agency in the public, commercial or not-for-profit sectors.

Competing interests None declared.

Patient consent for publication Not required.

Provenance and peer review Not commissioned; externally peer reviewed.

Data availability statement № data are available. As this is a protocol paper there are currently no data.
Open access This is an open access article distributed in accordance with the Creative Commons Attribution Non Commercial (CC BY-NC 4.0) license, which permits others to distribute, remix, adapt, build upon this work non-commercially, and license their derivative works on different terms, provided the original work is properly cited, appropriate credit is given, any changes made indicated, and the use is non-commercial. See: http://creativecommons.org/licenses/by-nc/4.0/.

ORCID iD

Moira Smith http://orcid.org/0000-0003-2085-7522

\section{REFERENCES}

1 Smith M, Neibling B, Williams G, et al. A qualitative study of active participation in sport and exercise for individuals with multiple sclerosis. Physiother Res Int 2019;24:e1776.

2 Smith M, Barker R, Williams G, et al. The effect of exercise on high-level mobility in individuals with neurodegenerative disease: a systematic literature review. Physiotherapy 2020;106:174-93.

3 Kjølhede T, Vissing K, de Place L, et al. Neuromuscular adaptations to long-term progressive resistance training translates to improved functional capacity for people with multiple sclerosis and is maintained at follow-up. Mult Scler 2015;21:599-611.

4 Latimer-Cheung AE, Pilutti LA, Hicks AL, et al. Effects of exercise training on fitness, mobility, fatigue, and health-related quality of life among adults with multiple sclerosis: a systematic review to inform Guideline development. Arch Phys Med Rehabil 2013;94:1800-28.

5 Gunn H, Markevics S, Haas B, et al. Systematic review: the effectiveness of interventions to reduce falls and improve balance in adults with multiple sclerosis. Arch Phys Med Rehabil 2015;96:1898-912.

6 Learmonth YC, Ensari I, MotI RW. Physiotherapy and walking outcomes in adults with multiple sclerosis: systematic review and meta-analysis. Physical Therapy Reviews 2016;21:160-72.

7 Pearson M, Dieberg G, Smart N. Exercise as a therapy for improvement of walking ability in adults with multiple sclerosis: a meta-analysis. Arch Phys Med Rehabil 2015;96:1339-48.

$8 \mathrm{Kim}$ Y, Lai B, Mehta T, et al. Exercise training guidelines for multiple sclerosis, stroke, and Parkinson disease: rapid review and synthesis. Am J Phys Med Rehabil 2019;98:613-21.

9 InAmerican College of Sports Medicine. ACSM's guidelines for exercise testing and prescription 10th. Philadelphia, Pennsylvania: Wolters Kluwer Health, 2018.

10 Australian Government Department of Health. Australia's physical activity and sedentary behaviour guidelines for adults (18-64 years), 2019. Available: https://www.health.gov.au/internet/main/publishing. nsf/Content/health-pubhlth-strateg-phys-act-guidelines2019

11 Thomas S, Kersten P, Thomas PW, et al. Exploring strategies used following a group-based fatigue management programme for people with multiple sclerosis (facets) via the fatigue management strategies questionnaire (FMSQ). BMJ Open 2015;5:e008274.

12 Michie S, Ashford S, Sniehotta FF, et al. A refined taxonomy of behaviour change techniques to help people change their physical activity and healthy eating behaviours: the CALO-RE taxonomy. Psychol Health 2011;26:1479-98.

13 Klaren RE, Motl RW, Dlugonski D, et al. Objectively quantified physical activity in persons with multiple sclerosis. Arch Phys Med Rehabil 2013;94:2342-8.

14 Latimer-Cheung AE, Martin Ginis KA, Hicks AL, et al. Development of evidence-informed physical activity guidelines for adults with multiple sclerosis. Arch Phys Med Rehabil 2013;94:1829-36.

15 Petajan JH, Gappmaier E, White AT, et al. Impact of aerobic training on fitness and quality of life in multiple sclerosis. Ann Neurol 1996;39:432-41.

16 Pilutti LA, Greenlee TA, Motl RW, et al. Effects of exercise training on fatigue in multiple sclerosis: a meta-analysis. Psychosom Med 2013;75:575-80.

17 Blikman LJ, Huisstede BM, Kooijmans $\mathrm{H}$, et al. Effectiveness of energy conservation treatment in reducing fatigue in multiple sclerosis: a systematic review and meta-analysis. Arch Phys Med Rehabil 2013;94:1360-76.

18 Andreasen AK, Stenager E, Dalgas U. The effect of exercise therapy on fatigue in multiple sclerosis. Mult Scler 2011;17:1041-54.

19 Platta ME, Ensari I, Motl RW, et al. Effect of exercise training on fitness in multiple sclerosis: a meta-analysis. Arch Phys Med Rehabil 2016;97:1564-72.

20 Paltamaa J, Sjögren T, Peurala SH, et al. Effects of physiotherapy interventions on balance in multiple sclerosis: a systematic review and meta-analysis of randomized controlled trials. J Rehabil Med 2012;44:811-23. 
21 Kjølhede T, Vissing K, Dalgas U. Multiple sclerosis and progressive resistance training: a systematic review. Mult Scler 2012;18:1215-28.

22 White LJ, Castellano V. Exercise and brain health--implications for multiple sclerosis: Part 1--neuronal growth factors. Sports Med 2008;38:91-100.

23 Kjølhede T, Siemonsen S, Wenzel D, et al. Can resistance training impact MRI outcomes in relapsing-remitting multiple sclerosis? Mult Scler 2018;24:1356-65.

24 Briken S, Rosenkranz SC, Keminer O, et al. Effects of exercise on irisin, BDNF and IL-6 serum levels in patients with progressive multiple sclerosis. J Neuroimmunol 2016;299:53-8.

25 Campos C, Rocha NBF, Lattari E, et al. Exercise-Induced neuroprotective effects on neurodegenerative diseases: the key role of trophic factors. Expert Rev Neurother 2016;16:723-34.

26 Mokhtarzade M, Ranjbar R, Majdinasab N, et al. Effect of aerobic interval training on serum IL-10, TNF $\alpha$, and adipokines levels in women with multiple sclerosis: possible relations with fatigue and quality of life. Endocrine 2017;57:262-71.

27 Deckx N, Wens I, Nuyts AH, et al. 12 weeks of combined endurance and resistance training reduces innate markers of inflammation in a randomized controlled clinical trial in patients with multiple sclerosis. Mediators Inflamm 2016;2016:1-13.

28 White LJ, Castellano V. Exercise and brain health--implications for multiple sclerosis: Part Il--immune factors and stress hormones. Sports Med 2008;38:179-86.

29 Negaresh R, Motl RW, Mokhtarzade M, et al. Effects of exercise training on cytokines and adipokines in multiple sclerosis: a systematic review. Mult Scler Relat Disord 2018;24:91-100.

30 Kjølhede T, Dalgas U, Gade AB, et al. Acute and chronic cytokine responses to resistance exercise and training in people with multiple sclerosis. Scand J Med Sci Sports 2016:26:824-34.

31 InCreswell JW, Plano Clark VL. Designing and conducting mixed methods research. Third ed. London;Thousand Oaks, California;New Delhi;Singapore: SAGE, 2018.

32 Thompson AJ, Banwell BL, Barkhof F, et al. Diagnosis of multiple sclerosis: 2017 revisions of the McDonald criteria. Lancet Neurol 2018:17:162-73.

33 Kurtzke JF. Rating neurologic impairment in multiple sclerosis: an expanded disability status scale (EDSS). Neurology 1983;33:1444-52.

34 Lublin FD. New multiple sclerosis phenotypic classification. Eur Neurol 2014;72 Suppl 1:1-5.

35 Julious SA. Sample size of 12 per group rule of thumb for a pilot study. Pharm Stat 2005;4:287-91.

36 Bowling A. Just one question: if one question works, why ask several? J Epidemiol Community Health 2005;59:342-5.

37 Learmonth YC, Motl RW. Important considerations for feasibility studies in physical activity research involving persons with multiple sclerosis: a scoping systematic review and case study. Pilot Feasibility Stud 2018:4:1.

38 Learmonth YC, Adamson BC, Kinnett-Hopkins D, et al. Results of a feasibility randomised controlled study of the guidelines for exercise in multiple sclerosis project. Contemp Clin Trials 2017:54:84-97.

39 Adamson BC, Learmonth YC, Kinnett-Hopkins D, et al. Feasibility study design and methods for project gems: guidelines for exercise in multiple sclerosis. Contemp Clin Trials 2016;47:32-9 https://doi. org/
40 World Health Organisation. International classification of functioning, disability and health (ICF), 2001. Available: www.who.int/ classifications/icf/en/ [Accessed 02.04.2019].

41 Kiresuk TJ, Sherman RE. Goal attainment scaling: a general method for evaluating comprehensive community mental health programs. Community Ment Health J 1968;4:443-53.

42 Turner-Stokes L. Goal attainment scaling (gas) in rehabilitation: a practical guide. Clin Rehabil 2009;23:362-70.

43 Khan F, Pallant JF, Turner-Stokes L. Use of goal attainment scaling in inpatient rehabilitation for persons with multiple sclerosis. Arch Phys Med Rehabil 2008;89:652-9.

44 Bovend'Eerdt TJH, Botell RE, Wade DT. Writing smart rehabilitation goals and achieving goal attainment scaling: a practical guide. Clin Rehabil 2009;23:352-61.

45 Kierkegaard M, Lundberg IE, Olsson T, et al. High-intensity resistance training in multiple sclerosis - An exploratory study of effects on immune markers in blood and cerebrospinal fluid, and on mood, fatigue, health-related quality of life, muscle strength, walking and cognition. J Neurol Sci 2016;362:251-7.

46 Zhou X, Fragala MS, McElhaney JE, et al. Conceptual and methodological issues relevant to cytokine and inflammatory marker measurements in clinical research. Curr Opin Clin Nutr Metab Care 2010;13:541-7.

47 Bostic TJ, McGartland Rubio D, Hood M. A validation of the subjective vitality scale using structural equation modeling. Soc Indic Res 2000;52:313-24.

48 Dawes H, Collett J, Meaney A, et al. Delayed recovery of leg fatigue symptoms following a maximal exercise session in people with multiple sclerosis. Neurorehabil Neural Repair 2014;28:139-48.

49 Williams G, Morris ME, Greenwood BN, et al. The high-level mobility assessment tool for traumatic brain injury: user manual. Melbourne: La Trobe University, 2004

50 Williams GP, Greenwood KM, Robertson VJ, et al. High-Level mobility assessment tool (HiMAT): interrater reliability, retest reliability, and internal consistency. Phys Ther 2006;86:395-400.

51 Aminian S, Motl RW, Rowley J, et al. Management of multiple sclerosis symptoms through reductions in sedentary behaviour: protocol for a feasibility study. BMJ Open 2019;9:e026622.

52 Qualtrics XM. Qualtrics, 2019. Available: https://www.qualtrics.com/ au/ [Accessed 14.06.19 2019].

53 Sandelowski M. What's in a name? qualitative description revisited. Res Nurs Health 2010;33:77-84

54 Stanley M. Qualitative descriptive a very good place to start. In: Nayer S, Stanley MD, eds. Qualitative research methodologies for occupational science and therapy. Oxon: Routledge, 2015.

55 Thabane L, Ma J, Chu R, et al. A tutorial on pilot studies: the what, why and how. BMC Med Res Methodol 2010;10:1.

56 Saldana J. The coding manual for qualitative researchers. 2nd. London England: SAGE, 2013.

57 Braun V, Clarke V. Using thematic analysis in psychology. Qual Res Psychol 2006;3:77-101.

58 Braun V, Clarke V. Successful qualitative research a practical guide for beginners. London, England: SAGE Publications Ltd, 2013.

59 National Health and Medical Research Council. National statement on ethical conduct in human research, 2018. Available: https:// www.nhmrc.gov.au/guidelines-publications/e72 [Accessed 02 Nov 17]. 